\title{
Implications of the Symptom-Level Overlap Among DSM Diagnoses for Dimensions of Psychopathology
}

\author{
Miriam K. Forbes, $\mathrm{PhD}$
}

Centre for Emotional Health, School of Psychological Sciences,

Macquarie University, Sydney, Australia

\author{
Address correspondence to: $\quad$ Miriam K. Forbes, $\mathrm{PhD}$ \\ Centre for Emotional Health, \\ School of Psychological Sciences, \\ Macquarie University, \\ Sydney, NSW, Australia, 2109 \\ Email: miri.forbes@mq.edu.au \\ Phone: +61298509436
}

Acknowledgements: I would like to thank the students who worked on extracting the unique constituent symptoms in each DSM-5 diagnosis and coding symptom-level redundancy: Anton Harris, Katherine Faure, Brier Michelsen, and Hannah Woodbridge. Thanks also to Robert F. Krueger, Ashley L. Watts, David Preece (handling editor), and anonymous reviewers at Journal of Emotion and Psychopathology for their invaluable feedback on earlier drafts of this paper.

I am a member of the Hierarchical Taxonomy of Psychopathology (HiTOP) Consortium and Executive Board. 


\begin{abstract}
Research on the patterns of covariation among mental disorders has proliferated, as summarized in the Hierarchical Taxonomy of Psychopathology (HiTOP). The aim of this brief descriptive study was to examine whether the repetition of symptoms among DSM-5 diagnoses is likely to be inflating the surface similarity of diagnoses in a way that artificially reinforces the dimensions that emerge when modelling patterns of disorder covariation or comorbidity. Specifically, the symptoms comprising the DSM-5 diagnostic criteria for all disorders covered by the HiTOP framework were examined for patterns of overlap that mirror the patterns of disorder covariation captured in HiTOP dimensions. I found that 358 pairs of the DSM-5 diagnoses covered by the HiTOP framework had one or more overlapping symptoms in their diagnostic criteria, and that a third $(\mathrm{n}=130 ; 34 \%)$ of the unique constituent symptoms reinforced the higher-order structure of HiTOP through repetition within dimensions and/or between dimensions in the same superspectrum. By contrast, $86 \%$ of the possible pairs of diagnoses did not have any shared symptoms, and the majority of the symptoms $(n=222 ; 58 \%)$ did not influence the structure through repetition. Further, a fifth $(n=71$; $19 \%$ ) of the symptoms worked against the HiTOP structure by increasing the surface similarity of diagnoses under different subfactors, spectra, and superspectra. Overall, while patterns of symptom-level overlap do not appear strong enough to account for the emergence of HiTOP dimensions, these patterns do seem likely to inflate the similarity and consequent covariation of some DSM-5 diagnoses. Research on the statistical structure of psychopathology that uses DSM-5 diagnostic constructs should account for this potential source of bias.
\end{abstract}

Key words: Hierarchical Taxonomy of Psychopathology (HiTOP); DSM-5; psychopathology; nosology; classification 


\section{Implications of the Symptom-Level Overlap Among DSM Diagnoses for Dimensions of Psychopathology}

In some of the earliest work on the statistical structure of common mental disorders, Krueger (1999) analysed the patterns of comorbidity among 10 mental disorders in the National Comorbidity Survey $(n=8098)$. Krueger found that several latent variables parsimoniously accounted for these patterns: Internalizing — bifurcated into Fear and Distress ${ }^{1}$ — and Externalizing. These dimensions formed the foundation for the subsequent proliferation of research on the statistical structure of psychopathology in adults, which is summarized in the Hierarchical Taxonomy of Psychopathology (HiTOP; Kotov et al., 2017, 2021; see Figure 1).

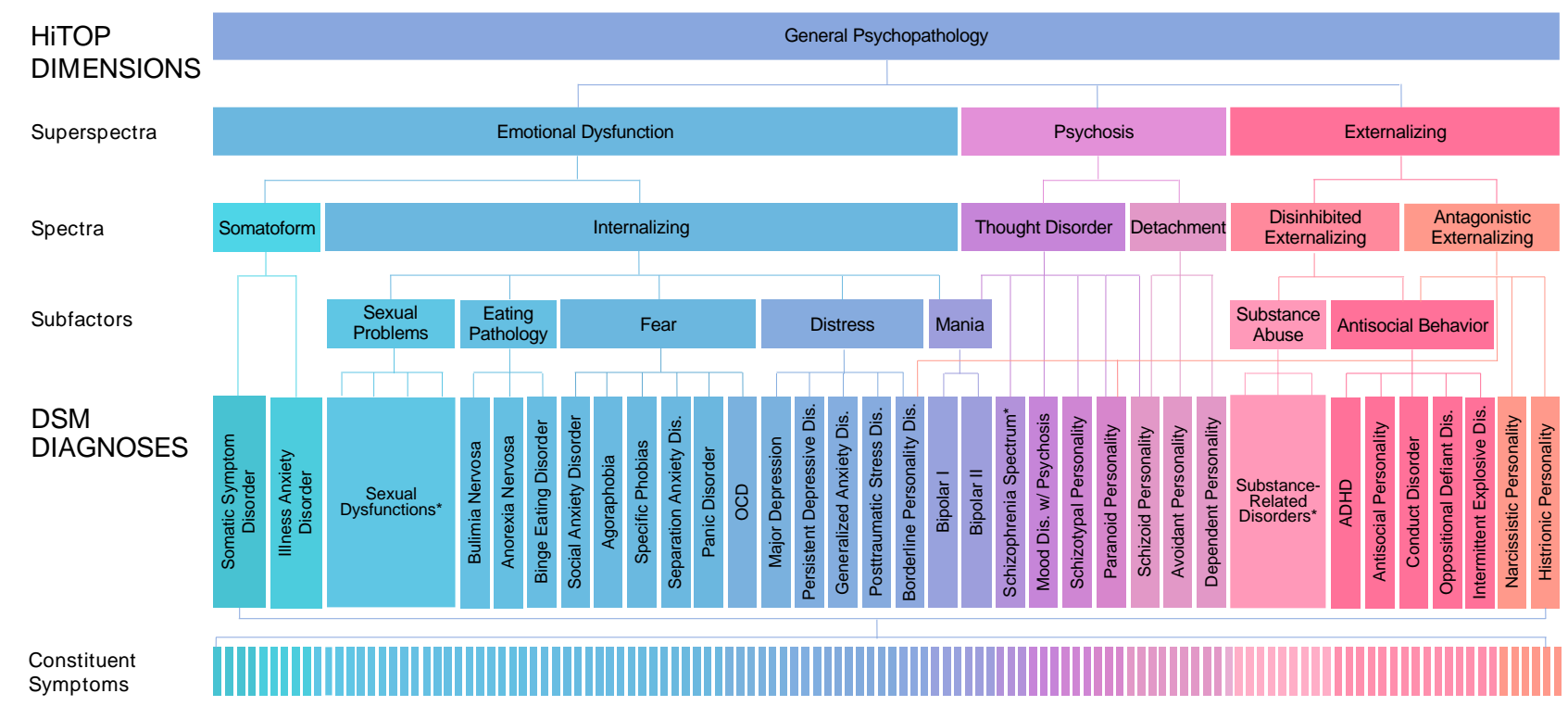

Figure 1. Dimensions in the Hierarchical Taxonomy of Psychopathology (HiTOP) framework-including the hypothesized superspectra of Emotional Dysfunction, Psychosis, and Externalizing (Kotov et al., 2021) - and the constituent DSM-5 diagnoses used in the present analyses. A negative association between Histrionic Personality Disorder and Detachment is not shown or included in analyses. Asterisks denote whole chapters of DSM-5 diagnoses.

However, from the outset of this literature, researchers have raised the concern that symptom-level overlap among DSM diagnoses could account for the emergent dimensions of psychopathology (e.g., Borsboom, 2002;

Wittchen et al., 1999). For example, in a commentary on Krueger (1999), Borsboom hypothesized:

"Some disorders (e.g., dysthymia and depression) have a number of criteria in common, whereas other disorders (e.g., dysthymia and social phobia) do not. If diagnoses are made without hierarchical exclusion rules, disorders having more criteria in common will tend to covary more than disorders having fewer criteria in common. However, finding this does not provide "new" empirical information; it is a direct result of the way the DSM is structured. In other words, given the DSM, these are the results you will find.” (p. 569).

\footnotetext{
${ }^{1}$ Originally labelled Anxious Misery.
} 
Offering some support for this hypothesis, Borsboom found a Spearman correlation of $\rho=.62$ between A) the correlation coefficients among the 10 mental disorders reported in Krueger (1999) and B) the number of shared DSM criteria for each pair of disorders.

Borsboom's (2002) hypothesis that the dimensions of psychopathology underpinning the HiTOP framework are a foregone conclusion of the patterns of symptom-level overlap among DSM diagnoses paints a troubling picture. If this hypothesis were supported and extended beyond the foundational dimensions in Krueger (1999), it could substantially undermine the validity and utility of the HiTOP framework, which has largely been built on analyses of DSM diagnoses. Rather than summarising potentially important signals, the HiTOP dimensions would merely be reflecting idiosyncratic artefacts of the structure of the DSM. Notably, several studies have already established that this is likely not the case. Symptom-level overlap among DSM diagnoses cannot fully account for the dimensions that emerge in this research (e.g., Krueger 2002; Lahey et al., 2018). For example, in response to Borsboom (2002), Krueger noted that the majority $(73 \%)$ of the correlations among the 10 mental disorders were unaffected by shared criteria, including all of the disorders indicating the Externalizing dimension.

However, it is not an all-or-nothing question whether shared diagnostic criteria between diagnoses could increase the surface similarity of disorders, potentially inflating the patterns of covariation and comorbidity captured by the dimensions in the HiTOP framework and artefactually reinforcing the likelihood of HiTOP subfactors, spectra, and/or superspectra emerging in analyses of these constructs (see Figure 1). Here I sought to understand to what extent the patterns of symptom-level overlap among DSM-5 diagnoses mirror the HiTOP dimensions, and to consider the implications of symptom overlap for each dimension.

\section{Method}

\section{Symptom coding}

The DSM-5 (American Psychiatric Association, 2013) diagnostic criteria for each mental disorder covered by the HiTOP framework (Figure 1) were the focus of these descriptive analyses. The unique constituent symptoms for each diagnosis were extracted from the manual using methods similar to Borsboom et al. (2011): Frequency, duration, severity, and causal specifications were removed from diagnostic criteria — as were distress and impairment criteria— to represent symptoms at their base level; disjunctive symptoms were separated into their constituent parts (e.g., insomnia or hypersomnia was coded as two separate symptoms); and symptoms that exclusively applied to children were removed. Symptoms were not permitted to repeat within diagnoses (e.g., psychomotor agitation is a symptom of both mania and depression in Bipolar I Disorder, but it was only included once in the list of constituent symptoms for Bipolar I). This process resulted in a detailed list of the symptoms comprising each diagnosis. For example, major depressive disorder had 21 symptoms, anorexia nervosa had seven, and panic disorder had 23. 
These symptoms were then coded for overlap between diagnoses. First, identical and nearly identical symptoms - for example, with minor grammatical differences_-were coded as the same symptom by assigning the same symptom reference number to each instance. All symptoms were then coded into content categories (i.e., subdomains of affective, behavioural, cognitive, and somatic symptoms) and screened for similar content. Symptoms deemed conceptually redundant (i.e., different phrasing of the same subjective experience) were also coded as the same symptom by assigning the same symptom reference number to each of them. For example, easily fatigued, fatigue or loss of energy, low energy or fatigue, fatigue, and lethargy were identified as one symptom repeating across multiple diagnoses. The full spreadsheet of all constituent symptoms and the redundancy coding between diagnoses is included in the supplementary materials for this project on the Open Science Framework (https://osf.io/kuacv/).

Symptoms nested within diagnoses were then coded for which HiTOP dimension(s) they fell under in Figure 1. Where diagnoses cross-loaded on two HiTOP dimensions (e.g., Borderline Personality Disorder cross-loads on Distress and Antagonistic Externalizing), the constituent symptoms were included in the analyses under both dimensions to capture the resulting similarity both within and between dimensions. Overall, the coding and analyses erred on the side of capturing all symptom-level overlap, some of which would not affect patterns of comorbidity, as discussed below.

\section{Analytic approach}

All analyses were descriptive. The focus was on identifying patterns of symptom overlap between diagnoses within the same HiTOP dimensions, which could artificially reinforce the dimensions. Patterns of symptom overlap between diagnoses under different dimensions were also examined, as these patterns would have the opposite effect but their absence could potentially add to the effect of symptom overlap within dimensions. The results are reported as proportions of the total number of unique symptoms in the list (i.e., with distinct symptom reference numbers), and as proportions of the unique symptoms within each dimension. A Sankey diagram (Figure 2) summarises the patterns of overlap at the level of the HiTOP dimension most proximal to each diagnosis in Figure 1. In this diagram, each symptom reference number is represented by a circle, the size of the circle represents the number of times that symptom repeated between diagnoses, and the lines connect the symptom to the HiTOP dimension(s) in which it occurs. Line saturation and style are used to contrast the patterns of overlap between diagnoses in the same HiTOP dimensions (left of Figure 2) versus in different HiTOP dimensions (right of Figure 2). Finally, a Spearman correlation was calculated between A) the meta-analytic correlation coefficients for 22 of the diagnoses from a recent meta-analysis of structural evidence for the HiTOP framework (Ringwald et al., 2021) and B) the number of shared symptoms for each pair of these diagnoses, for comparison with Borsboom (2002).

\section{Results}

The 71 DSM-5 diagnoses and specifiers captured in the HiTOP framework comprised 382 distinct symptoms; 358 pairs of diagnoses had one or more overlapping symptoms (14\% of all possible pairs of diagnoses). Figure 2 shows 
how the patterns of overlap among the symptoms mapped onto the HiTOP dimensions. In total—including the repetition that arose due to diagnoses cross-loading onto more than one dimension-222 (58\%) of the symptoms were part of the criteria for only a single diagnosis and did not repeat within or between HiTOP dimensions. As shown on the left of Figure 2, 118 symptoms (31\%) repeated in two or more diagnoses under the same HiTOP dimension; on the right of Figure 2, 89 symptoms (23\%) repeated in diagnoses under different dimensions. The proportions of symptom overlap within and between the dimensions in Figure 1 are also given in Table 1.

Overlap within dimensions was calculated as a percentage of the symptoms within a dimension that were part of the diagnostic criteria for two or more diagnoses in that dimension. Detachment, Antagonistic Externalizing, and Fear had no symptom repetition; Thought Disorder, Antisocial Behavior, Distress, Eating Pathology, Sexual Problems, and Somatoform had some (11-26\%) symptom repetition; and Substance Use and Mania had the majority of symptoms (53\% and $100 \%$, respectively) overlapping across multiple disorders within the dimension.

Overlap between dimensions was calculated as a percentage of the unique symptoms within each dimension that were also part of the unique symptoms in another dimension: Eating Pathology, Sexual Problems, and Somatoform had no symptom overlap with other dimensions; Detachment, Antisocial Behavior, Substance Use, and Fear had some (18-42\%) overlap with other dimensions; and Thought Disorder, Antagonistic Externalizing, Mania, and Distress had the majority of symptoms (54-78\%) overlapping with other dimensions. The pairwise patterns of overlap are discussed in more detail below.

Taking the higher-order structure of HiTOP into account (see Figure 1$)$, half ( $n=44 ; 49 \%$ ) of the 89 symptoms that repeated between different dimensions included repetitions within the same superspectrum, and most $(n=71 ; 80 \%)$ included repetitions between dimensions in different superspectra.

Overall, a third ( $n=130 ; 34 \%$ ) of the 382 distinct symptoms reinforced the higher-order structure of HiTOP through repetition within dimensions and/or between dimensions in the same superspectrum, whereas the majority of symptoms $(n=222 ; 58 \%)$ did not influence the structure through repetition, and a fifth $(n=71 ; 19 \%)$ worked against the structure at the subfactor, spectrum, and superspectrum level. ${ }^{2}$

Finally, the Spearman correlation between A) the meta-analytic correlation coefficients for 22 of the diagnoses from a recent meta-analysis of structural evidence for the HiTOP framework (Ringwald et al., 2021) and B) the number of shared symptoms for each pair of these diagnoses was $\rho=.28$ (95\% CI .15-.40).

\footnotetext{
${ }^{2}$ These numbers do not necessarily sum to $100 \%$ because symptoms can both reinforce and work against the structure.
} 
SYMPTOM OVERLAP BETWEEN DIAGNOSES IN THE SAME DIMENSION
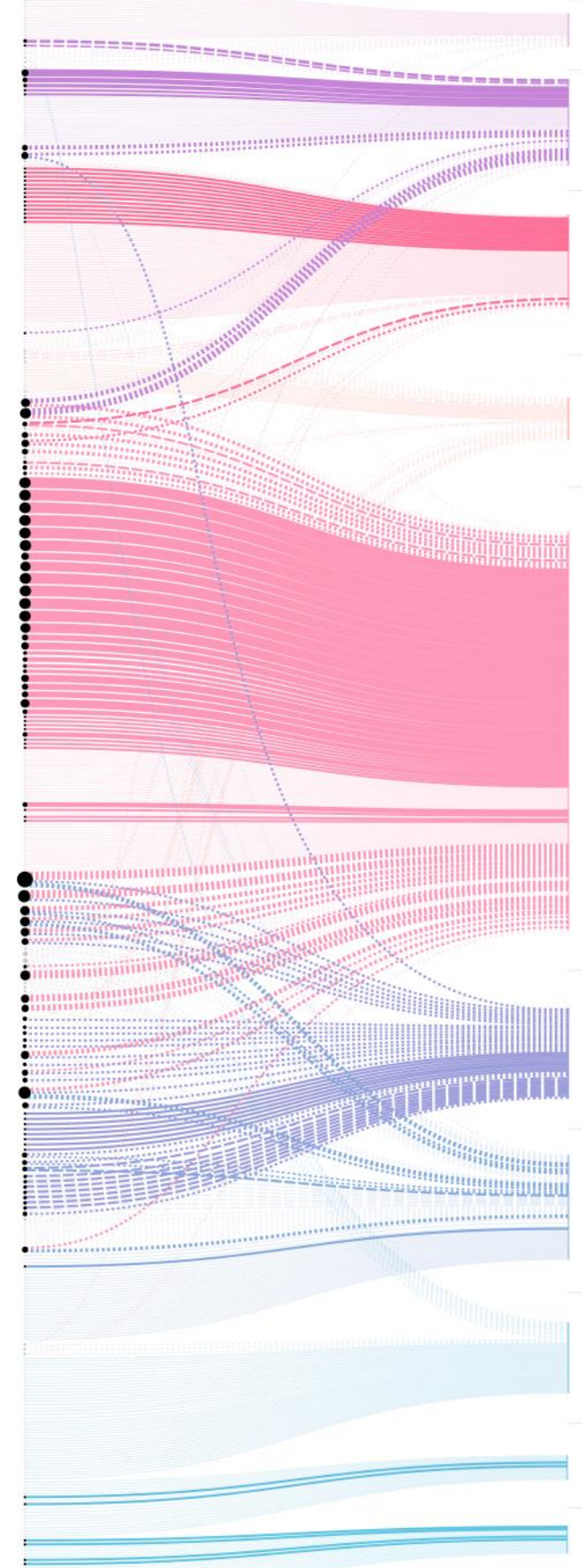

HITOP DIMENSIONS $n(\%)$ overlap within | between

Detachment
$0 / 31(0 \%) \mid 13 / 31(42 \%)$

Thought Disorder 12/50 (24\%) | 27/50 (54\%)

\section{Antisocial Behavior}

$6 / 61(26 \%) \mid 11 / 61(18 \%)$

\section{Antagonistic Externalizing} $0 / 44(0 \%) \mid 34 / 44(77 \%)$

\section{Substance Use} 59/111 (53\%) |39/111 (35\%

Mania 37/37 (100\%) | 29/37 (78\%)

Distress 10/73 (14\%) | 44/73(60\%)

Fear $0 / 56(0 \%)$ | 15/56 (27\%)

\section{Eating Pathology 2/18 (11\%) | 0/18 (0\%) \\ Sexual Problems 4/18 (22\%) | 0/18 (0\%) $\underset{1 / 6(17 \%) \mid 0 / 6(0 \%)}{\text { Somatoform }}$}

SYMPTOM OVERLAP BETWEEN DIAGNOSES IN DIFFERENT DIMENSIONS 


\begin{tabular}{|c|c|c|c|c|c|c|c|c|c|c|c|}
\hline & Detachment & $\begin{array}{l}\text { Thought } \\
\text { Disorder }\end{array}$ & $\begin{array}{l}\text { Antisocial } \\
\text { Behavior }\end{array}$ & $\begin{array}{l}\text { Antagonistic } \\
\text { Externalizing }\end{array}$ & Substance Use & Mania & Distress & Fear & $\begin{array}{l}\text { Eating } \\
\text { Pathology }\end{array}$ & $\begin{array}{c}\text { Sexual } \\
\text { Problems }\end{array}$ & Somatoform \\
\hline Detachment & 31 & $18.0 \%$ & $1.6 \%$ & $0.0 \%$ & $0.9 \%$ & $5.4 \%$ & $4.1 \%$ & $5.4 \%$ & $0.0 \%$ & $0.0 \%$ & $0.0 \%$ \\
\hline Thought Disorder & $29.0 \%$ & 50 & $1.6 \%$ & $29.5 \%$ & $7.2 \%$ & $2.7 \%$ & $8.2 \%$ & $5.4 \%$ & $0.0 \%$ & $0.0 \%$ & $0.0 \%$ \\
\hline Antisocial Behavior & $3.2 \%$ & $2.0 \%$ & 61 & $6.8 \%$ & $5.4 \%$ & $5.4 \%$ & $6.8 \%$ & $0.0 \%$ & $0.0 \%$ & $0.0 \%$ & $0.0 \%$ \\
\hline Antagonistic Externalizing & $0.0 \%$ & $26.0 \%$ & $4.9 \%$ & 44 & $5.4 \%$ & $16.2 \%$ & $24.7 \%$ & $3.6 \%$ & $0.0 \%$ & $0.0 \%$ & $0.0 \%$ \\
\hline Substance Use & $3.2 \%$ & $16.0 \%$ & $9.8 \%$ & $13.6 \%$ & 111 & $32.4 \%$ & $27.4 \%$ & $19.6 \%$ & $0.0 \%$ & $0.0 \%$ & $0.0 \%$ \\
\hline Mania & $6.5 \%$ & $2.0 \%$ & $3.3 \%$ & $13.6 \%$ & $10.8 \%$ & 37 & $30.1 \%$ & $0.0 \%$ & $0.0 \%$ & $0.0 \%$ & $0.0 \%$ \\
\hline Distress & $9.7 \%$ & $12.0 \%$ & $8.2 \%$ & $40.9 \%$ & $18.0 \%$ & $59.5 \%$ & $\mathbf{7 3}$ & $3.6 \%$ & $0.0 \%$ & $0.0 \%$ & $0.0 \%$ \\
\hline Fear & $9.7 \%$ & $6.0 \%$ & $0.0 \%$ & $4.5 \%$ & $9.9 \%$ & $0.0 \%$ & $2.7 \%$ & 56 & $0.0 \%$ & $0.0 \%$ & $0.0 \%$ \\
\hline Eating Pathology & $0.0 \%$ & $0.0 \%$ & $0.0 \%$ & $0.0 \%$ & $0.0 \%$ & $0.0 \%$ & $0.0 \%$ & $0.0 \%$ & 18 & $0.0 \%$ & $0.0 \%$ \\
\hline Sexual Problems & $0.0 \%$ & $0.0 \%$ & $0.0 \%$ & $0.0 \%$ & $0.0 \%$ & $0.0 \%$ & $0.0 \%$ & $0.0 \%$ & $0.0 \%$ & 18 & $0.0 \%$ \\
\hline Somatoform & $0.0 \%$ & $0.0 \%$ & $0.0 \%$ & $0.0 \%$ & $0.0 \%$ & $0.0 \%$ & $0.0 \%$ & $0.0 \%$ & $0.0 \%$ & $0.0 \%$ & 6 \\
\hline $\begin{array}{l}\text { Total (Overlap with any } \\
\text { other dimension) }\end{array}$ & $41.9 \%$ & $54.0 \%$ & $18.0 \%$ & $77.3 \%$ & $35.1 \%$ & $78.4 \%$ & $60.3 \%$ & $26.8 \%$ & $0.0 \%$ & $0.0 \%$ & $0.0 \%$ \\
\hline $\begin{array}{l}\text { Overlap among diagnoses } \\
\text { within the dimension }\end{array}$ & $0.0 \%$ & $24.0 \%$ & $26.2 \%$ & $0.0 \%$ & $53.2 \%$ & $100.0 \%$ & $13.7 \%$ & $0.0 \%$ & $11.1 \%$ & $22.2 \%$ & $16.7 \%$ \\
\hline
\end{tabular}

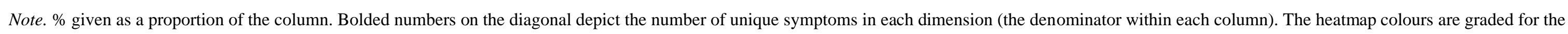

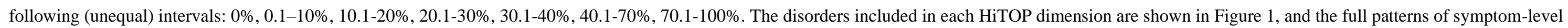
overlap are shown in Figure 2. 


\section{Discussion}

This study examined whether symptom-level overlap within and between DSM diagnoses comprising the HiTOP dimensions (Figure 1) is likely to represent an important source of bias in the literature underpinning the HiTOP framework due to inflating the surface similarity of the constructs being studied. There was evidence of potential bias for some dimensions, as discussed below, and substantial variability in the proportion of symptoms overlapping within and between dimensions (Figure 2 and Table 1).

Evidence for systematic patterns of symptom overlap within HiTOP dimensions would be the most likely to bias research on the structure of psychopathology because it would suggest the patterns of covariation among disorders - and latent variables summarising this information - are an artefact of their surface phenomenological similarity, rather than deeper mechanisms or processes of interest (cf. Borsboom, 2002). Levels of within-dimension symptom overlap varied substantially. For example, Fear, Antagonistic Externalizing, and Detachment had no symptom repetition among their constituent diagnoses, whereas for Substance Use and Mania the majority of symptoms repeated in multiple diagnoses. Notably, for Substance Use, these patterns of symptom overlap are unlikely to confound research focusing on comorbidity among these disorders because symptoms of Substance Use Disorders are contingent of the use of specific substances. So while subsets of Substance Use Disorders may be more likely to co-occur as a result of their shared relationship with a specific substance (e.g., Cannabis Intoxication, Cannabis Use Disorder, and Cannabis Withdrawal - all related to cannabis use), the fact that psychomotor retardation is a symptom for both Opioid Intoxication and Stimulant Withdrawal, for example, seems unlikely to artificially inflate comorbidity.

By contrast, when ignoring hierarchical exclusion rules to better understand the natural structure of psychopathology (Kotov et al., 2018), the symptom overlap within the Mania, Antisocial Behavior, and Thought Disorder spectra is more likely to inflate the lifetime patterns of comorbidity among those disorders. For example, Bipolar I and Bipolar II share 100\% of their symptoms, and Conduct Disorder and Antisocial Personality Disorder share many symptoms, such as unlawful behaviours, deceitfulness, and lack of remorse. Similarly, all of the Schizophrenia Spectrum diagnoses in the DSM-5 share the key features of delusions, hallucinations, disorganised thinking and speech, grossly disorganised or abnormal motor behavior, and negative symptoms such as flattened affect, avolition, alogia, anhedonia, and asociality (American Psychiatric Association, 2013). These similarities mean that diagnoses made without hierarchical exclusion rules may be likely to overestimate comorbidity purely based on phenomenological similarity. Distress also had some noteworthy internal repetition of symptoms like difficulty concentrating, irritability, insomnia, and fatigue. However, both Thought Disorder and Distress had less within-dimension symptom overlap than between-dimension overlap. 
Symptom-level overlap was less of a concern in other dimensions. For example, Somatic Symptom Disorder and Illness Anxiety Disorder — constituting the Somatoform spectrum—shared only anxiety about health as a symptom; Eating Pathology diagnoses had two overlapping symptoms (undue influence of body weight or shape, and binge eating); and the shared symptoms among Sexual Problems diagnoses were between mutually exclusive diagnoses by sex (e.g., Female Orgasmic Disorder and [male] Delayed Ejaculation).

The patterns of overlap between HiTOP dimensions largely reflected the duplicated symptom lists included in analyses for disorders that cross-loaded on multiple dimensions-for example, Borderline Personality Disorder crossloads on Antagonstic Externalizing and Distress; Schizoid Personality Disorder cross-loads on Detachment and Thought Disorder; and Paranoid Personality Disorder cross-loads on Antagonistic Externalizing and Thought Disorder. Similarly, the presence of Major Depressive Episode criteria in both Mania and Distress accounted for most of the overlap between those dimensions. A more interesting pattern of results was evident in the symptom overlap of Substance Use with Mania, Distress, and (to a lesser extent) Fear: Many Substance Use Disorders include hallmark symptoms of mania, depression, and anxiety (e.g., fatigue, irritability, insomnia and hypersomnia, psychomotor agitation and retardation, increases and decreases in appetite, elevated mood, inexhaustibility, difficulty concentrating, hypervigilance, and physical anxiety symptoms such as palpitations, nausea, and dizziness, among others). These patterns of overlap have interesting potential implications whereby apparent symptoms of substance intoxication or withdrawal could be due to co-occurring internalizing psychopathology, or vice versa. Notably, these patterns of symptom overlap work against the HiTOP structure — increasing the surface similarity of diagnoses in different dimensions - rather than reinforcing it.

Finally, when examining the correspondence between the number of overlapping symptoms and the metaanalytic correlations between diagnoses reported in Ringwald et al. (2021), the Spearman correlation found here was less than half the strength of the correlation Borsboom (2002) found for the Krueger (1999) results (i.e., $\rho=.28$, compared to $\rho=.62$ ). This suggests that the patterns of disorder covariation that underpin HiTOP dimensions reflect other mechanisms beyond shared phenomenology. For example, many of the dimensions have been found to account for shared environmental risks as well as shared cognitive, genetic, and other biological vulnerabilities, supporting the validity and utility of these dimensions (Kotov et al., 2021).

\section{Limitations}

It is important to reiterate that this was a purely descriptive study. The goal was simply to quantify the symptom-level overlap among diagnoses captured by the HiTOP framework. Further research will be needed to test the extent to which accounting for symptom overlap might shape or change the statistical structure of HiTOP dimensions that are derived based on patterns of covariation or comorbidity among DSM diagnoses (e.g., Lahey et al., 2018). Further, the coding of symptom overlap in this study was liberal, aiming to capture all instances of repetition, including 
when diagnoses were part of more than one HiTOP dimension and when the symptom overlap would be unlikely to affect patterns of comorbidity. For example, delay in orgasm is a symptom shared by Female Orgasmic Disorder and [male] Delayed Ejaculation; because these disorders are mutually exclusive, relying on the notion of a gender binary, this surface similarity could only increase the likelihood of both diagnoses being given to one individual if they were received before and after a gender transition. As above, statistical modelling of how symptom overlap changes dimensions of psychopathology would be the best way to quantify these effects.

\section{Conclusion}

In sum, the results found here suggest that symptom-level homogeneity likely inflates the similarity and consequent covariation of some DSM-5 disorders that is captured in HiTOP dimensions. Symptom-level overlap thus represents a potential source of bias in the literature underpinning the HiTOP model (i.e., Kotov et al., 2017). However, the patterns of overlap among symptoms certainly do not appear systematic enough to make the whole HiTOP structure a foregone conclusion (cf. Borsboom, 2002): Most symptoms (58\%) were specific to a single diagnosis covered by the HiTOP framework, and a substantial minority of symptoms (19\%) worked against the HiTOP structure by increasing the surface similarity of diagnoses under different subfactors, spectra, and superspectra.

Regardless, research on the statistical structure of psychopathology should be aware of this potential source of bias if analysing variables based on DSM diagnoses. Better yet, research aiming to operationalise HiTOP dimensions should move beyond using DSM diagnoses — for example, using measures that are well-suited to measuring HiTOP dimensions (see https://hitop.unt.edu/clinical-tools/hitop-friendly-measures) or symptom-level data (e.g., Forbes et al., 2021) would both circumvent issues related to symptom overlap among traditional diagnostic categories. This shift in measurement approaches will become even easier as new measures become available for assessing the full HiTOP framework (e.g., Simms et al., 2020), and time will tell how much these changes in measurement will affect our understanding of the statistical structure of psychopathology. 


\section{References}

American Psychiatric Association. (2013). Schizophrenia Spectrum and Other Psychotic Disorders. In Diagnostic and statistical manual of mental disorders (5th ed.). https://doi.org/10.1176/appi.books.9780890425596.dsm02

Borsboom, D. (2002). The structure of the DSM. Archives of General Psychiatry, 59, 569-570. https://doi.org/10.1001/archpsyc.59.6.569

Borsboom, D., Cramer, A. O., Schmittmann, V. D., Epskamp, S., \& Waldorp, L. J. (2011). The small world of psychopathology. PloS One, 6(11), e27407. https://doi.org/10.1371/journal.pone.0027407

Forbes, M. K., Sunderland, M., Rapee, R. M., Batterham, P. J., Calear, A. L., Carragher, N., ... \& Krueger, R. F. (2021). A detailed hierarchical model of psychopathology: From individual symptoms up to the general factor of psychopathology. Clinical Psychological Science, 9(2), 139-168. https://doi.org/10.1177\%2F2167702620954799

Kotov, R., Krueger, R. F., Watson, D., Achenbach, T. M., Althoff, R. R., Bagby, R. M., Brown, T. A., Carpenter, W. T., Caspi, A., Clark, L. A., Eaton, N. R., Forbes, M. K., Forbush, K. T., Goldberg, D., Hasin, D., Hyman, S. E., Ivanova, M. Y., Lynam, D. R., Markon, K., . . Zimmerman, M. (2017). The Hierarchical Taxonomy of Psychopathology (HiTOP): A dimensional alternative to traditional nosologies. Journal of Abnormal Psychology, 126, 454-477. https://doi.org/10.1037/abn0000258

Kotov, R., Krueger, R. F., Watson, D., Cicero, D. C., Conway, C. C., DeYoung, C. G., Eaton, N. R., Forbes, M. K., Hallquist, M. N., Latzman, R. D., Mullins-Sweatt, S. N., Ruggero, C. J., Simms, L. J., Waldman, I. D., Waszczuk, M. A., \& Wright, A. G. (2021). The Hierarchical Taxonomy of Psychopathology (HiTOP): A quantitative nosology based on consensus of evidence. Annual Review of Clinical Psychology, 17, 83-108. https://doi.org/10.1146/annurevclinpsy-081219-093304

Kotov, R., Ruggero, C. J., Krueger, R. F., Watson, D., \& Zimmerman, M. (2018). The perils of hierarchical exclusion rules: A further word of caution. Depression and Anxiety, 9, 903-904. https://doi.org/10.1002/da.22826

Krueger R. F. (1999). The structure of common mental disorders. Archives of General Psychiatry, 56, 921-6. https://doi.org/10.1001/archpsyc.56.10.921

Krueger R. F. (2002). The Structure of the DSM. Archives of General Psychiatry, 59, 570-1.

Lahey, B. B., Zald, D. H., Perkins, S. F., Villalta-Gil, V., Werts, K. B., Van Hulle, C. A., ... \& Waldman, I. D. (2018). Measuring the hierarchical general factor model of psychopathology in young adults. International Journal of Methods in Psychiatric Research, 27(1), e1593-600. https://doi.org/10.1002/mpr.1593

Ringwald, W. R., Forbes, M. K., \& Wright, A. G. (2021). Meta-analysis of structural evidence for the Hierarchical Taxonomy of Psychopathology (HiTOP) model. Psychological Medicine, 1-14. https://doi.org/10.1017/S0033291721001902

Simms, L. J., Wright, A. G., Cicero, D., Kotov, R., Mullins-Sweatt, S. N., Sellbom, M., ... \& Zimmermann, J. (2020). Development of measures for the Hierarchical Taxonomy of Psychopathology (HiTOP): A collaborative scale development project. Assessment. https://doi.org/10.1177\%2F10731911211015309

Wittchen, H. U., Höfler, M., \& Merikangas, K. (1999). Toward the identification of core psychopathological processes?. Archives of General Psychiatry, 56, 929-931. https://doi.org/10.1001/archpsyc.56.10.929 